\title{
If You Book It, Will They Come? Attendance at Postdischarge Follow-Up Visits Scheduled by Inpatient Providers
}

\author{
Rahul Banerjee, MD ${ }^{1 *}$, Alex Suarez, BS², Melanie Kier, BA², Steve Honeywell Jr., BS², Weiwei Feng, MS³, Nandita Mitra, PhD³, \\ David T. Grande, MD, MPA',2, Jennifer S. Myers, MD'1
}

${ }^{1}$ Department of Medicine, Hospital of the University of Pennsylvania, University of Pennsylvania, Philadelphia, Pennsylvania; ${ }^{2}$ Perelman School of Medicine, University of Pennsylvania, Philadelphia, Pennsylvania; ${ }^{3}$ Department of Biostatistics and Epidemiology, University of Pennsylvania, Philadelphia, Pennsylvania.

BACKGROUND: Postdischarge follow-up visits (PDFVs) are widely recommended to improve inpatient-outpatient transitions of care.

\section{OBJECTIVE: To measure PDFV attendance rates.}

DESIGN: Observational cohort study.

SETTING: Medical units at an academic quaternary-care hospital and its affiliated outpatient clinics.

PATIENTS: Adult patients hospitalized between April 2014 and March 2015 for whom at least 1 PDFV with our health system was scheduled. Exclusion criteria included nonprovider visits, visits cancelled before discharge, nonaccepted health insurance, and visits scheduled for deceased patients.

MEASUREMENTS: The study outcome was the incidence of PDFVs resulting in no-shows or same-day cancellations (NS/SDCs).
RESULTS: Of all hospitalizations, 6136 (52\%) with 9258 PDFVs were analyzed. Twenty-five percent of PDFVs were NS/ SDCs, $23 \%$ were cancelled before the visit, and $52 \%$ were attended as scheduled. In multivariable regression models, NS/SDC risk factors included black race (odds ratio [OR] 1.94, 95\% confidence interval [Cl], 1.63-2.32), longer lengths of stay (hospitalizations $\geq 15$ days: OR $1.51,95 \% \mathrm{Cl}, 1.22-$ 1.88), and discharge to facility (OR 2.10, 95\% Cl, 1.70-2.60). Conversely, NS/SDC visits were less likely with advancing age (age $\geq 65$ years: OR $0.39,95 \% \mathrm{Cl}, 0.31-0.49$ ) and driving distance (highest quartile: OR $0.65,95 \% \mathrm{Cl}, 0.52-0.81$ ). Primary care visits had higher NS/SDC rates (OR 2.62, 95\% CI, 2.03-3.38) than oncologic visits. The time interval between discharge and PDFV was not associated with NS/SDC rates.

CONCLUSIONS: PDFVs were scheduled for more than half of hospitalizations, but $25 \%$ resulted in NS/SDCs. New strategies are needed to improve PDFV attendance. Journal of Hospital Medicine 2017;12:618-625. (C) 2017 Society of Hospital Medicine
Given growing incentives to reduce readmission rates, predischarge checklists and bundles have recommended that inpatient providers schedule postdischarge follow-up visits (PDFVs) for their hospitalized patients. ${ }^{1-4}$ PDFVs have been linked to lower readmission rates in patients with chronic conditions, including congestive heart failure, psychiatric illnesses, and chronic obstructive pulmonary disease. ${ }^{5-8}$ In contrast, the impact of PDFVs on readmissions in hospitalized general medicine populations has been mixed.9-12 Beyond the presence or absence of PDFVs, it may be a patient's inability to keep scheduled PDFVs that contributes more strongly to preventable readmissions. ${ }^{11}$

This challenge, dealing with the $12 \%$ to $37 \%$ of patients who miss their visits ("no-shows"), is not new. ${ }^{13-17}$ In highrisk patient populations, such as those with substance abuse,

\footnotetext{
*Address for correspondence and reprint requests: Rahul Banerjee, MD, Department of Medicine, Hospital of the University of Pennsylvania, 3400 Spruce St, 100 Centrex, Philadelphia, PA 19104; Telephone: 267-303-7995; Fax: 215662-7919; E-mail: rahul.banerjee.md@gmail.com
}

Additional Supporting Information may be found in the online version of this article.

Received: November 21, 2016; Revised: February 27, 2016; Accepted: March 19, 2017

2017 Society of Hospital Medicine DOI 10.12788/jhm.2777 diabetes, or human immunodeficiency virus, no-shows (NSs) have been linked to poorer short-term and long-term clinical outcomes. ${ }^{16,18-20}$ Additionally, NSs pose a challenge for outpatient clinics and the healthcare system at large. The financial cost of NSs ranges from approximately $\$ 200$ per patient in 2 analyses to $\$ 7$ million in cumulative lost revenue per year at 1 large academic health system. ${ }^{13,17,21}$ As such, increasing attendance at PDFVs is a potential target for improving both patient outcomes and clinic productivity.

Most prior PDFV research has focused on readmission risk rather than PDFV attendance as the primary outcome. ${ }^{5-12}$ However, given the patient-oriented benefits of attending PDFVs and the clinic-oriented benefits of avoiding vacant time slots, NS PDFVs represent an important missed opportunity for our healthcare delivery system. To our knowledge, risk factors for PDFV nonattendance have not yet been systematically studied. The aim of our study was to analyze PDFV nonattendance, particularly NSs and same-day cancellations (SDCs), for hospitalizations and clinics within our healthcare system.

\section{METHODS}

\section{Study Design}

We conducted an observational cohort study of adult patients from 10 medical units at the Hospital of the University 
TABLE 1. Baseline Patient, Hospitalization, and PDFV Characteristics

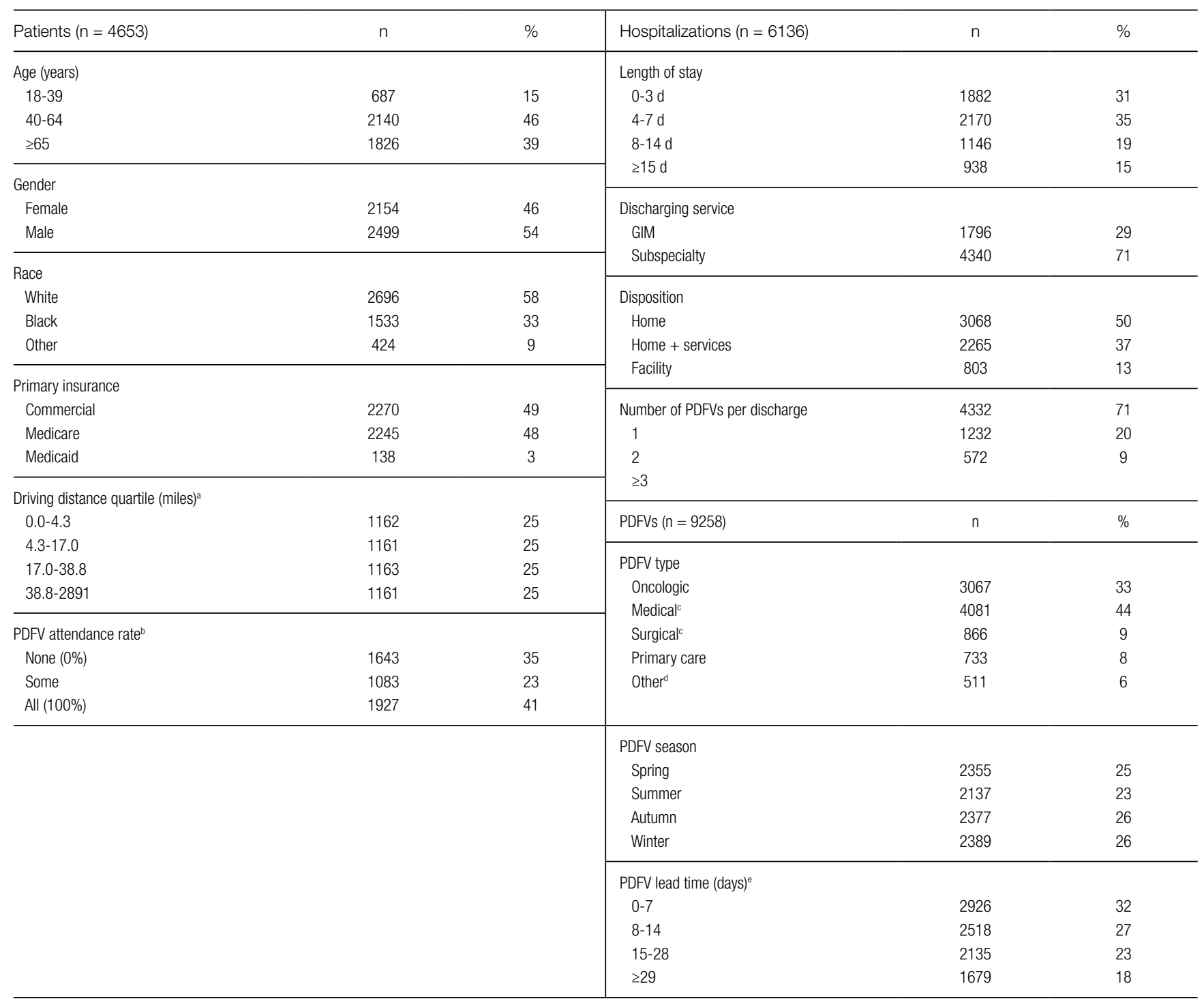

aDriving distances for 6 patients $(0.13 \%)$ were unavailable.

'PDFV attendance-as-scheduled rate for a given patient. See Supplementary Figure A for additional details.

'Apart from medical oncology, surgical oncology, or radiation oncology.

¿See Supplementary Table B for PDFV frequencies broken down by type of clinic.

'The number of days between discharge and the appointment.

NOTE: Abbreviation: GIM, general internal medicine; PDFV, postdischarge follow-up visit.

of Pennsylvania (a 789-bed quaternary-care hospital within an urban, academic medical system) who were scheduled with at least 1 PDFV. Specifically, the patients included in our analysis were hospitalized on general internal medicine services or medical subspecialty services with discharge dates between April 1, 2014, and March 31, 2015. Hospitalizations included in our study had at least 1 PDFV scheduled with an outpatient provider affiliated with the University of Pennsylvania Health System (UPHS). PDFVs scheduled with unaffiliated providers were not examined.

Each PDFV was requested by a patient's inpatient care team. Once the care team had determined that a PDFV was clinically warranted, a member of the team (generally a resident, advanced practice provider, medical student, or designee) either called the UPHS clinic to schedule an appointment time or e-mailed the outpatient UPHS provider directly to facilitate a more urgent PDFV appointment time. Once a PDFV time was confirmed, PDFV details (ie, date, time, location, and phone number) were electronically entered into the patient's discharge instructions by the inpatient care team. At the time of discharge, nurses reviewed these instructions with their patients. All patients left the hospital with a physical copy of these instructions. As part of routine care at our institution, patients then received au- 
TABLE 2. PDFV Nonattendance By Patient and Hospitalization Characteristics

\begin{tabular}{|c|c|c|c|c|c|}
\hline \multirow[b]{2}{*}{ Visit Characteristic } & \multirow{2}{*}{$\begin{array}{c}\text { Total } \\
\mathrm{n}\end{array}$} & \multicolumn{2}{|c|}{ NS/SDC } & \multirow[b]{2}{*}{ Adjusted ORs } & \multirow[b]{2}{*}{$95 \% \mathrm{Cl}$} \\
\hline & & $\%$ & & & \\
\hline \multicolumn{6}{|l|}{ Patient age (years) } \\
\hline $18-39$ & 1416 & 494 & 35 & 1.00 & - \\
\hline v40-64 & 4360 & 1083 & 25 & $0.63^{\mathrm{a}}$ & $0.52-0.76$ \\
\hline$\geq 65$ & 3482 & 726 & 21 & $0.39^{\mathrm{a}}$ & $0.31-0.49$ \\
\hline \multicolumn{6}{|l|}{ Patient gender } \\
\hline Female & 4279 & 1176 & 27 & 1.00 & - \\
\hline Male & 4979 & 1127 & 23 & 0.97 & $0.85-1.10$ \\
\hline \multicolumn{6}{|l|}{ Patient race } \\
\hline White & 5349 & 953 & 18 & 1.00 & - \\
\hline Black & 3056 & 1180 & 39 & $1.94^{\mathrm{a}}$ & $1.63-2.32$ \\
\hline Other & 853 & 170 & 20 & 1.04 & $0.82-1.33$ \\
\hline \multicolumn{6}{|c|}{ Patient primary insurance } \\
\hline Commercial & 4553 & 1070 & 24 & 1.00 & - \\
\hline Medicare & 4385 & 1131 & 26 & 1.25 & $0.88-1.78$ \\
\hline Medicaid & 320 & 102 & 32 & $1.41^{\mathrm{a}}$ & $1.19-1.67$ \\
\hline \multicolumn{6}{|c|}{ Driving distance quartiles (miles) ${ }^{\mathrm{b}}$} \\
\hline $0-4.3$ & 2314 & 851 & 37 & 1.00 & - \\
\hline $4.3-17.0$ & 2335 & 631 & 27 & 0.84 & $0.70-1.01$ \\
\hline $17.0-38.8$ & 2428 & 426 & 18 & $0.67^{\mathrm{a}}$ & $0.54-0.83$ \\
\hline $38.8-2891$ & 2164 & 393 & 18 & $0.65^{\mathrm{a}}$ & $0.52-0.81$ \\
\hline \multicolumn{6}{|l|}{ Length of stay (days) } \\
\hline $0-3$ & 2497 & 517 & 21 & 1.00 & - \\
\hline $4-7$ & 3230 & 812 & 25 & $1.24^{\mathrm{a}}$ & $1.05-1.46$ \\
\hline $8-14$ & 1985 & 540 & 27 & $1.29^{\mathrm{a}}$ & $1.06-1.57$ \\
\hline$\geq 15$ & 1546 & 434 & 28 & $1.51^{\mathrm{a}}$ & $1.22-1.88$ \\
\hline \multicolumn{6}{|l|}{ Discharging service } \\
\hline GIM & 2653 & 953 & 36 & 1.00 & - \\
\hline Subspecialty & 6605 & 1350 & 20 & $0.79^{\mathrm{a}}$ & $0.68-0.93$ \\
\hline \multicolumn{6}{|l|}{ Disposition } \\
\hline Home & 4216 & 873 & 21 & 1.00 & - \\
\hline Home + services & 3637 & 985 & 27 & $1.32^{\mathrm{a}}$ & $1.01-1.36$ \\
\hline Facility & 1405 & 445 & 32 & $2.10^{\mathrm{a}}$ & $1.70-2.60$ \\
\hline \multicolumn{6}{|c|}{ Number of PDFVs per discharge } \\
\hline 1 & 4332 & 1023 & 24 & 1.00 & - \\
\hline 2 & 2464 & 756 & 31 & $1.17^{\mathrm{a}}$ & $1.01-1.36$ \\
\hline$\geq 3$ & 2462 & 524 & 21 & $0.82^{\mathrm{a}}$ & $0.69-0.98$ \\
\hline
\end{tabular}

a Statistically significant with $\alpha=0.05$.

${ }^{\mathrm{b}}$ Driving distances for 17 visits $(0.18 \%)$ were unavailable.

NOTE: Abbreviations: Cl, confidence interval; GIM, general internal medicine; NS/SDC, no-show or same-day cancellation; OR, odds ratio; PDFV, postdischarge follow-up visit.

tomated telephone reminders from their UPHS-affiliated outpatient clinic 48 hours prior to each PDFV.

\section{Data Collection}

Our study was determined to meet criteria for quality improvement by the University of Pennsylvania's Institutional Review Board. We used our healthcare system's integrated electronic medical record system to track the dates of initial PDFV requests, the dates of hospitalization, and actual PDFV dates. PDFVs were included if the appointment request was made while a patient was hospitalized, including the day of dis- charge. Our study methodology only allowed us to investigate PDFVs scheduled with UPHS outpatient providers. We did not review discharge instructions or survey non-UPHS clinics to quantify visits scheduled with other providers, for example, community health centers or external private practices.

Exclusion criteria included the following: (1) office visits with nonproviders, for example, scheduled diagnostic procedures or pharmacist appointments for warfarin dosing; (2) visits cancelled by inpatient providers prior to discharge; (3) visits for patients not otherwise eligible for UPHS outpatient care because of insurance reasons; and (4) visits scheduled 


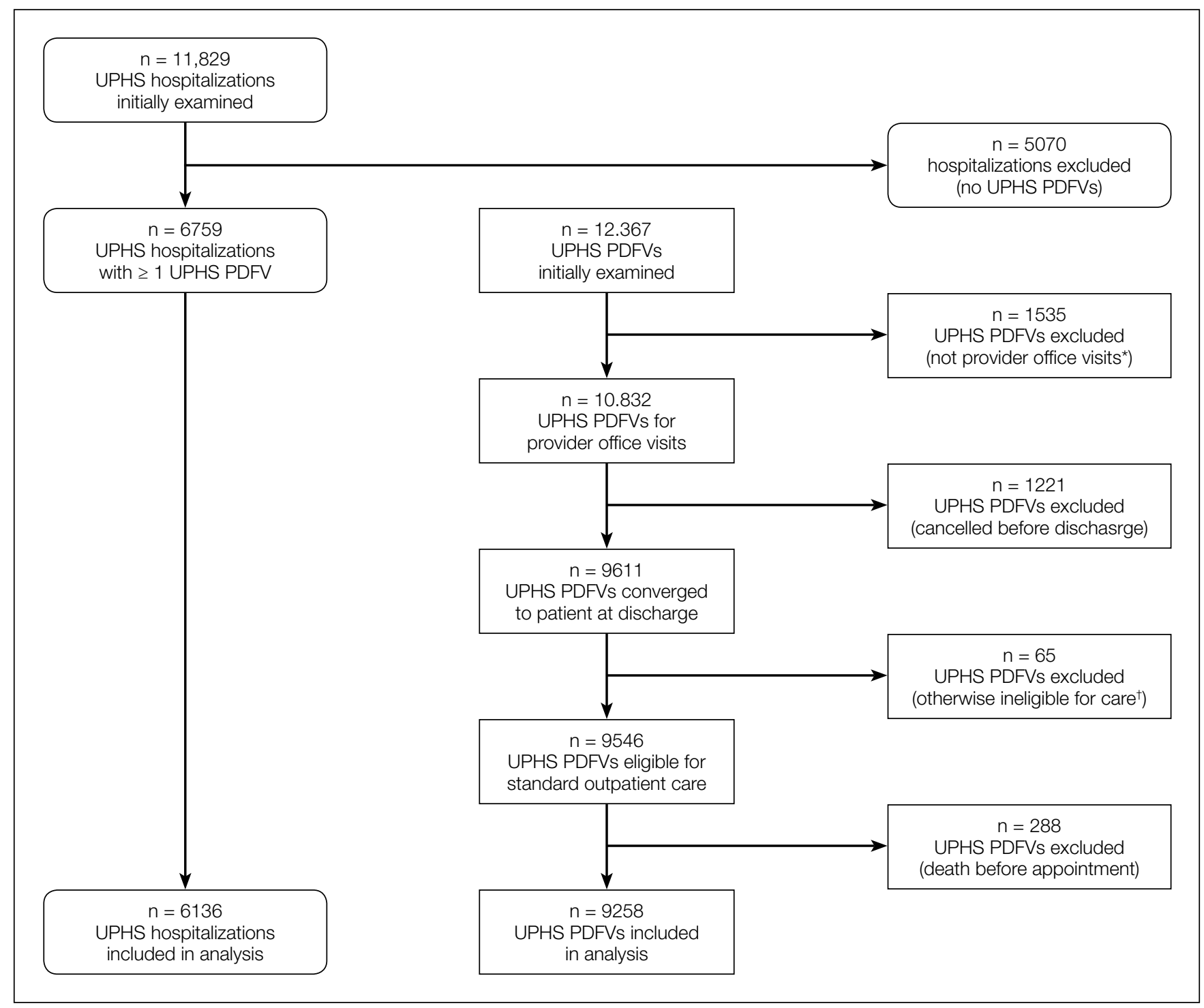

FIG. Flow chart of inclusion and exclusion criteria. Only hospitalizations and PDFVs within this health system could be examined using our study methodology. A) For example: chemotherapy infusions, diagnostic procedures, and pharmacist visits for warfarin dosing. B) Appointments scheduled for patients ( $n=47$ ) who would otherwise be ineligible for UPHS outpatient care for insurance reasons; see Supplementary Table A for details.

for dates after a patient's death. Our motivation for the third exclusion criterion was the infrequent and irregular process by which PDFVs were authorized for these patients. These patients and their characteristics are described in Supplementary Table 1 in more detail.

For each PDFV, we recorded age, gender, race, insurance status, driving distance, length of stay for index hospitalization, discharging service (general internal medicine vs subspecialty), postdischarge disposition (home, home with home care services such as nursing or physical therapy, or facility), the number of PDFVs scheduled per index hospitalization, PDFV specialty type (oncologic subspecialty, nononcologic medical subspecialty, nononcologic surgical subspecialty, primary care, or other specialty), PDFV season, and PDFV lead time (the number of days between the discharge date and PDFV). We consolidated oncologic specialties into 1 group given the integrated nature of our healthcare system's comprehensive cancer center. "Other" PDFV specialty subtypes are described in Supplementary Table 2. Driving distances between patient postal codes and our hospital were calculated using Excel VBA Master (Salt Lake City, Utah) and were subsequently categorized into patient-level quartiles for further analysis. For cancelled PDFVs, we collected dates of cancellation relative to the date of the appointment itself.

\section{Study Outcomes}

The primary study outcome was PDFV attendance. Each PDFV's status was categorized by outpatient clinic staff as attended, cancelled, or NS. For cancelled appointments, cancellation dates and reasons (if entered by clinic representatives) were collected. In keeping with prior studies investigating outpatient nonattendance,we calculated collective 
TABLE 3. PDFV Nonattendance By Appointment Characteristics

\begin{tabular}{|c|c|c|c|c|c|}
\hline \multirow[b]{2}{*}{ Visit characteristic } & \multirow[b]{2}{*}{ Total } & \multicolumn{2}{|c|}{ NS/SDC } & \multirow[b]{2}{*}{ Adjusted ORs } & \multirow[b]{2}{*}{$95 \% \mathrm{Cl}$} \\
\hline & & $\mathrm{n}$ & $\%$ & & \\
\hline \multicolumn{6}{|l|}{ PDFV type } \\
\hline Oncologic & 3067 & 354 & 12 & 1.00 & - \\
\hline Medical $^{\mathrm{a}}$ & 4081 & 1188 & 29 & $2.31^{\mathrm{b}}$ & $1.93-2.76$ \\
\hline Surgical ${ }^{\mathrm{a}}$ & 866 & 265 & 31 & $1.98^{\mathrm{b}}$ & $1.56-2.52$ \\
\hline Primary care & 733 & 286 & 39 & $2.62^{\mathrm{b}}$ & $2.03-3.38$ \\
\hline Other ${ }^{c}$ & 511 & 210 & 41 & $3.44 b$ & $2.60-4.54$ \\
\hline \multicolumn{6}{|l|}{ PDFV season } \\
\hline Spring & 2355 & 574 & 24 & 1.00 & - \\
\hline Summer & 2137 & 462 & 22 & $0.81^{\mathrm{b}}$ & $0.68-0.97$ \\
\hline Autumn & 2377 & 623 & 26 & 1.05 & $0.88-1.25$ \\
\hline Winter & 2389 & 644 & 27 & 1.15 & $0.96-1.36$ \\
\hline \multicolumn{6}{|l|}{ PDFV lead time (days) ${ }^{d}$} \\
\hline $0-7$ & 2926 & 641 & 22 & 1.00 & - \\
\hline $8-14$ & 2518 & 644 & 26 & 1.04 & $0.89-1.22$ \\
\hline $15-28$ & 2135 & 597 & 28 & 1.05 & $0.88-1.24$ \\
\hline$\geq 29$ & 1679 & 421 & 25 & 0.96 & $0.79-1.16$ \\
\hline \multicolumn{6}{|c|}{ aApart from medical oncology, surgical oncology, or radiation oncology. } \\
\hline \multicolumn{6}{|c|}{ 'Statistically significant with $\alpha=0.05$. } \\
\hline \multicolumn{6}{|c|}{ 'See Supplementary Table B for NS/SDC rates broken down by type of clinic. } \\
\hline \multicolumn{6}{|c|}{ The number of days between discharge and the appointment. } \\
\hline
\end{tabular}

NS/SDC rates for the variables listed above. ${ }^{17,22-25} \mathrm{We}$ additionally calculated NS/SDC and attendance-as-scheduled rates stratified by the number of PDFVs per patient to assess for a "high-utilizer" effect with regard to PDFV attendance.

\section{Statistical Analysis}

We used multivariable mixed-effects regression with a logit link to assess associations between age, gender, race, insurance, driving distance quartile, length of stay, discharging service, postdischarge disposition, the number of PDFVs per hospitalization, PDFV specialty type, PDFV season, PDFV lead time, and our NS/SDC outcome. The mixed-effects approach was used to account for correlation structures induced by patients who had multiple visits and for patients with multiple hospitalizations. Specifically, our model specified 2 levels of nesting (PDFVs nested within each hospitalization, which were nested within each patient) to obtain appropriate standard error estimates for our adjusted odds ratios (ORs). Correlation matrices and multivariable variance inflation factors were used to assess collinearity among the predictor variables. These assessments did not indicate strong collinearity; hence, all predictors were included in the model. Only driving distance had a small amount of missing data $(0.18 \%$ of driving distances were unavailable), so multiple imputation was not undertaken. Analyses were performed using R version 3.3.1 (R Foundation for Statistical Computing, Vienna, Austria).

\section{RESULTS}

\section{Baseline Characteristics}

During the 1-year study period, there were 11,829 discrete hospitalizations in medical units at our hospital. Of these hospitalizations, 6136 (52\%) had at least 1 UPHS-affiliated PDFV meeting our inclusion and exclusion criteria, as detailed in the Figure. Across these hospitalizations, 9258 PDFVs were scheduled on behalf of 4653 patients. Demographic characteristics for these patients, hospitalizations, and visits are detailed in Table 1 . The median age of patients in our cohort was 61 years old (interquartile range [IQR] 49-70, range 18-101). The median driving distance was 17 miles (IQR 4.338.8, range 0-2891). For hospitalizations, the median length of stay was 5 days (IQR 3-10, range 0-97). The median PDFV lead time, which is defined as the number of days between discharge and PDFV, was 12 days (IQR 6-23, range 1-60). Overall, $41 \%$ of patients $(n=1927)$ attended all of their PDFVs as scheduled; Supplementary Figure 1 lists patient-level PDFV attendance-as-scheduled percentages in more detail.

\section{Incidence of NSs and SDCs}

Twenty-five percent of PDFVs ( $\mathrm{n}=2303$ ) were ultimately NS/SDCs; this included 1658 NSs (18\% of all appointments) and 645 SDCs (7\% of all appointments). Fifty-two percent of PDFVs $(n=4847)$ were kept as scheduled, while $23 \%(n=2108)$ were cancelled before the day of the visit. Of the 2558 cancellations with valid cancellation dates, $49 \%$ ( $n=1252$ ) were cancelled 2 or fewer days beforehand, as shown in Supplementary Figure 2.

In Table 2, we show unadjusted NS/SDC rates and adjusted NS/SDC ORs based on patient and hospitalization characteristics. NS/SDC appointments were more likely to occur in patients who were black (adjusted OR 1.94, 
95\% confidence interval [CI], 1.63-2.32) or Medicaid insured (OR 1.41, 95\% CI, 1.19-1.67). In contrast, NS/SDC appointments were less likely in elderly patients (age $\geq 65$ years: OR 0.39, 95\% CI, 0.31-0.49) and patients who lived further away (furthest quartile of driving distance: OR 0.65, 95\% CI, 0.52--0.81). Longer hospitalizations were associated with higher NS/SDC rates (length of stay $\geq 15$ days: OR 1.51, 95\% CI, 1.22-1.88). In contrast, discharges from subspecialty services (OR 0.79, 95\% CI, 0.68-0.93) had independently lower NS/SDC rates. Compared to discharges to home without services, NS/SDC rates were higher with discharges to home with services (OR 1.32, 95\% CI, 1.011.36) and with discharges to facilities (OR 2.10, 95\% CI, 1.70-2.60).

The presence of exactly $2 \mathrm{PDFV}$ s per hospitalization was also associated with higher NS/SDC rates (OR 1.17, 95\% CI, 1.01-1.36), compared to a single PDFV per hospitalization; however, the presence of more than 2 PDFVs per hospitalization was associated with lower NS/SDC rates (OR 0.82, 95\% CI, 0.69-0.98). A separate analysis (data not shown) of potential high utilizers revealed a $15 \% \mathrm{NS} / \mathrm{SDC}$ rate for the top $0.5 \%$ of patients (median: 18 PDFVs each) and an $18 \%$ NS/SDC rate for the top $1 \%$ of patients (median: 14 PDFVs each) with regard to the numbers of PDFVs scheduled, compared to the $25 \%$ overall NS/SDC rate for all patients.

NS/SDC rates and adjusted ORs with regard to individual PDFV characteristics are displayed in Table 3. Nononcologic visits had higher NS/SDC rates than oncologic visits; for example, the NS/SDC rate for primary care visits was 39\% (OR 2.62, 95\% CI, 2.03-3.38), compared to $12 \%$ for oncologic visits. Appointments in the "other" specialty category also had high nonattendance rates, as further described in Supplementary Table B. Summertime appointments were more likely to be attended (OR 0.81, 95\% CI, 0.68-0.97) compared to those in the spring. PDFV lead time (the time interval between the discharge date and appointment date) was not associated with changes in visit attendance.

\section{DISCUSSION}

PDFVs were scheduled on patients' behalf for more than half of all medical hospitalizations at our institution, a rate that is consistent with previous studies. ${ }^{10,11,26}$ However, 1 in 4 of these PDFVs resulted in a NS/SDC. This figure contrasts sharply with our institution's $10 \%$ overall NS/SDC rate for all outpatient visits (S. Schlegel, written communication, July 2016). In our study, patients who were younger, black, or Medicaid insured were more likely to miss their follow-up visits. Patients who lived farther from the study hospital had lower NS/SDC rates, which is consistent with another study of a low-income, urban patient population. ${ }^{27}$ In contrast, patients with longer lengths of stay, discharges with home care services, or discharges to another facility were more likely to miss their PDFVs. Reasons for this are likely multifactorial, including readmission to a hospital or feeling too unwell to leave home to attend PDFVs. Insurance policies regarding ambulance reimbursement and outpatient billing can cause confusion and may have contributed to higher NS/SDC rates for facility-bound patients. ${ }^{28,29}$

When comparing PDFV characteristics themselves, oncologic visits had the lowest NS/SDC incidence of any group analyzed in our study. This may be related to the inherent life-altering nature of a cancer diagnosis or our cancer center's use of patient navigators. ${ }^{23,30}$ In contrast, primary care clinics suffered from NS/SDC rates approaching 40\%, which is a concerning finding given the importance of primary care coordination in the posthospitalization period. ${ }^{9,31}$ Why are primary care appointments so commonly missed? Some studies suggest that forgetting about a primary care appointment is a leading reason. ${ }^{15,32,33}$ For PDFVs, this phenomenon may be augmented because the visits are not scheduled by patients themselves. Additionally, patients may paradoxically undervalue the benefit of an all-encompassing primary care visit, compared to a PDFV focused on a specific problem, (eg, a cardiology follow-up appointment for a patient with congestive heart failure). In particular, patients with limited health literacy may potentially undervalue the capabilities of their primary care clinics. ${ }^{34,35}$

The low absolute number of primary care PDFVs (only $8 \%$ of all visits) scheduled for patients at our hospital was an unexpected finding. This low percentage is likely a function of the patient population hospitalized at our large, urban quaternary-care facility. First, an unknown number of patients may have had PDFVs manually scheduled with primary care providers external to our health system; these PDFVs were not captured within our study. Second, $71 \%$ of the hospitalizations in our study occurred in subspecialty services, for which specific primary care follow-up may not be as urgent. Supporting this fact, further analysis of the 6136 hospitalizations in our study (data not shown) revealed that $28 \%$ of the hospitalizations in general internal medicine were scheduled with at least 1 primary care PDFV as opposed to only $5 \%$ of subspecialty-service hospitalizations.

In contrast to several previous studies of outpatient nonattendance,we did not find that visits scheduled for time points further in the future were more likely to be missed. . $^{14,24,25,36,37}$ Unlike other appointments, it may be that PDFV lead time does not affect attendance because of the unique manner in which PDFV times are scheduled and conveyed to patients. Unlike other appointments, patients do not schedule PDFVs themselves but instead learn about their PDFV dates as part of a large set of discharge instructions. This practice may result in poor recall of PDFV dates in recently hospitalized patient $s^{38}$, regardless of the lead time between discharge and the visit itself.

Supplementary Table 1 details a $51 \%$ NS/SDC rate for the small number of PDFVs $(n=65)$ that were excluded a priori from our analysis because of general ineligibility for UPHS outpatient care. We specifically chose to exclude this population because of the infrequent and irregular process by which these PDFVs were authorized on a case-by-case basis, typically via active engagement by our hospital's social work department. We did not study this population further but 
postulate that the $51 \% \mathrm{NS} / \mathrm{SDC}$ rate may reflect other social determinants of health that contribute to appointment nonadherence in a predominantly uninsured population.

Beyond their effect on patient outcomes, improving PDFV-related processes has the potential to boost both inpatient and outpatient provider satisfaction. From the standpoint of frontline inpatient providers (often resident physicians), calling outpatient clinics to request PDFVs is viewed as 1 of the top 5 administrative tasks that interfere with house staff education. ${ }^{39}$ Future interventions that involve patients in the PDFV scheduling process may improve inpatient workflow while simultaneously engaging patients in their own care. For example, asking clinic representatives to directly schedule PDFVs with hospitalized patients, either by phone or in person, has been shown in pilot studies to improve PDFV attendance and decrease readmissions. ${ }^{40-42}$ Conversely, NS/SDC visits harm outpatient provider productivity and decrease provider availability for other patients. ${ }^{13,17,43}$ Strategies to mitigate the impact of unfilled appointment slots (eg, deliberately overbooking time slots in advance) carry their own risks, including provider burnout. ${ }^{44}$ As such, preventing NSs may be superior to curing their adverse impacts. Many such strategies exist in the ambulatory setting, ${ }^{13,43,45}$ for example, better communication with patients through texting or goal-directed, personalized phone reminders. ${ }^{46-48}$

Our study methodology has several limitations. Most importantly, we were unable to measure PDFVs made with providers unaffiliated with UPHS. As previously noted, our low proportion of primary care PDFVs may specifically reflect patients with primary care providers outside of our health system. Similarly, our low percentage of Medicaid patients receiving PDFVs may be related to follow-up visits with nonaffiliated community health centers. We were unable to measure patient acuity and health literacy as potential predictors of NS/SDC rates. Driving distances were calculated from patient postal codes to our hospital, not to individual outpatient clinics. However, the majority of our hospital-affiliated clinics are located adjacent to our hospital; additionally, we grouped driving distances into quartiles for our analysis. We had initially attempted to differentiate between clinic-initiated and patient-initiated cancellations, but unfortunately, we found that the data were too unreliable to be used for further analysis (outlined in Supplementary Table 3). Lastly, because we studied patients in medical units at a single large, urban, academic center, our results are not generalizable to other settings (eg, community hospitals, hospitals with smaller networks of outpatient providers, or patients being discharged from surgical services or observation units).

\section{CONCLUSION}

Given national efforts to enhance postdischarge transitions of care, we aimed to analyze attendance at provider-scheduled PDFV appointments. Our finding that $25 \%$ of PDFVs resulted in NS/SDCs raises both questions and opportunities for inpatient and outpatient providers. Further research is needed to understand why so many patients miss their PD-
FVs, and we should work as a field to develop creative solutions to improve PDFV scheduling and attendance.

\section{Acknowledgments}

The authors acknowledge Marie Synnestvedt, $\mathrm{PhD}$, and Manik Chhabra, MD, for their assistance with data gathering and statistical analysis. They also acknowledge Allison DeKosky, MD, Michael Serpa, BS, Michael McFall, and Scott Schlegel, MBA, for their assistance with researching this topic. They did not receive external compensation for their assistance outside of their usual salary support.

Disclosure: Nothing to report.

\section{References}

1. Halasyamani L, Kripalani S, Coleman E, et al. Transition of care for hospitalized elderly patients - development of a discharge checklist for hospitalists. J Hosp Med. 2006;1(6):354-360.

2. Koehler BE, Richter KM, Youngblood L, et al. Reduction of 30-day postdischarge hospital readmission or emergency department (ED) visit rates in high-risk elderly medical patients through delivery of a targeted care bundle. J Hosp Med. 2009;4(4):211-218

3. Soong C, Daub S, Lee JG, et al. Development of a checklist of safe discharge practices for hospital patients. J Hosp Med. 2013;8(8):444-449.

4. Rice YB, Barnes CA, Rastogi R, Hillstrom TJ, Steinkeler CN. Tackling 30-day, all-cause readmissions with a patient-centered transitional care bundle. Popul Health Manag. 2016;19(1):56-62.

5. Nelson EA, Maruish MM, Axler JL. Effects of discharge planning and compliance with outpatient appointments on readmission rates. Psych Serv. 2000;51(7): 885-889.

6. Gavish R, Levy A, Dekel OK, Karp E, Maimon N. The association between hos pital readmission and pulmonologist follow-up visits in patients with chronic obstructive pulmonary disease. Chest. 2015;148(2):375-381.

7. Jackson C, Shahsahebi M, Wedlake T, DuBard CA. Timeliness of outpatient follow-up: an evidence-based approach for planning after hospital discharge. Ann Fam Med. 2015;13(2):115-122.

8. Donaho EK, Hall AC, Gass JA, et al. Protocol-driven allied health post-discharge transition clinic to reduce hospital readmissions in heart failure. J Am Heart Assoc. 2015;4(12):e002296

9. Misky GJ, Wald HL, Coleman EA. Post-hospitalization transitions: Examining the effects of timing of primary care provider follow-up. J Hosp Med. 2010;5(7): 392-397.

10. Grafft CA, McDonald FS, Ruud KL, Liesinger JT, Johnson MG, Naessens JM. Effect of hospital follow-up appointment on clinical event outcomes and mortality. Arch Intern Med. 2010;171(11):955-960.

11. Auerbach AD, Kripalani S, Vasilevskis EE, et al. Preventability and causes of readmissions in a national cohort of general medicine patients. JAMA Intern Med. 2016;176(4):484-493

12. Field TS, Ogarek J, Garber L, Reed G, Gurwitz JH. Association of early post-discharge follow-up by a primary care physician and 30-day rehospitalization among older adults. J Gen Intern Med. 2015;30(5):565-571

13. Quinn K. It's no-show time! Med Group Manage Assoc Connexion. 2007;7(6): 44-49.

14. Whittle J, Schectman G, Lu N, Baar B, Mayo-Smith MF. Relationship of scheduling interval to missed and cancelled clinic appointments. J Ambulatory Care Manage. 2008;31(4):290-302.

15. Kaplan-Lewis E, Percac-Lima S. No-show to primary care appointments: Why patients do not come. J Prim Care Community Health. 2013;4(4):251-255.

16. Molfenter T. Reducing appointment no-shows: Going from theory to practice. Subst Use Misuse. 2013;48(9):743-749.

17. Kheirkhah P, Feng Q, Travis LM, Tavakoli-Tabasi S, Sharafkhaneh A. Prevalence, predictors and economic consequences of no-shows. BMC Health Serv Res. 2016;16(1):13.

18. Colubi MM, Perez-Elias MJ, Elias L, et al. Missing scheduled visits in the outpatient clinic as a marker of short-term admissions and death. HIV Clin Trials. 2012;13(5):289-295

19. Obialo CI, Hunt WC, Bashir K, Zager PG. Relationship of missed and shortened hemodialysis treatments to hospitalization and mortality: Observations from a US dialysis network. Clin Kidney J. 2012;5(4):315-319.

20. Hwang AS, Atlas SJ, Cronin P, et al. Appointment "no-shows" are an independent predictor of subsequent quality of care and resource utilization outcomes. J Gen Intern Med. 2015;30(10):1426-1433.

21. Perez FD, Xie J, Sin A, et al. Characteristics and direct costs of academic pediatric 
subspecialty outpatient no-show events. J Healthc Qual. 2014;36(4):32-42.

22. Huang Y, Zuniga P. Effective cancellation policy to reduce the negative impact of patient no-show. Journal of the Operational Research Society. 2013;65(5):605-615.

23. Percac-Lima S, Cronin PR, Ryan DP, Chabner BA, Daly EA, Kimball AB. Patient navigation based on predictive modeling decreases no-show rates in cancer care. Cancer. 2015;121(10):1662-1670.

24. Torres O, Rothberg MB, Garb J, Ogunneye O, Onyema J, Higgins T. Risk factor model to predict a missed clinic appointment in an urban, academic, and underserved setting. Popul Health Manag. 2015;18(2):131-136.

25. Eid WE, Shehata SF, Cole DA, Doerman KL. Predictors of nonattendance at an endocrinology outpatient clinic. Endocr Pract. 2016;22(8):983-989.

26. Kashiwagi DT, Burton MC, Kirkland LL, Cha S, Varkey P. Do timely outpatient follow-up visits decrease hospital readmission rates? Am J Med Qual. 2012;27(1):11-15.

27. Miller AJ, Chae E, Peterson E, Ko AB. Predictors of repeated "no-showing" to clinic appointments. Am J Otolaryngol. 2015;36(3):411-414.

28. ASCO. Billing challenges for residents of Skilled Nursing Facilities. J Oncol Pract. 2008;4(5):245-248.

29. Centers for Medicare \& Medicaid Services (2013). "SE0433: Skilled Nursing Facility consolidated billing as it relates to ambulance services." Medicare Learning Network Matters. https://www.cms.gov/Outreach-and-Education/ Medicare-Learning-Network-MLN/MLNMattersArticles/downloads/se0433.pdf. Accessed on February 14, 2017.

30. Luckett R, Pena N, Vitonis A, Bernstein MR, Feldman S. Effect of patient navigator program on no-show rates at an academic referral colposcopy clinic. J Women Health (Larchmt). 2015;24(7):608-615.

31. Jones CD, Vu MB, O'Donnell CM, et al. A failure to communicate: A qualitative exploration of care coordination between hospitalists and primary care providers around patient hospitalizations. J Gen Intern Med. 2015;30(4):417-424.

32. George A, Rubin G. Non-attendance in general practice: a systematic review and its implications for access to primary health care. Fam Pract. 2003;20(2):178-184.

33. Samuels RC, Ward VL, Melvin P, et al. Missed Appointments: Factors Contrib uting to High No-Show Rates in an Urban Pediatrics Primary Care Clinic. Clin Pediatr (Phila). 2015;54(10):976-982.

34. Kangovi S, Barg FK, Carter T, Long JA, Shannon R, Grande D. Understanding why patients of low socioeconomic status prefer hospitals over ambulatory care. Health Aff (Millwood). 2013;32(7):1196-1203.
35. Long T, Genao I, Horwitz LI. Reasons for readmission in an underserved high-risk population: A qualitative analysis of a series of inpatient interviews. BMJ Open. 2013;3(9):e003212.

36. Lee VJ, Earnest A, Chen MI, Krishnan B. Predictors of failed attendances in a multi-specialty outpatient centre using electronic databases. BMC Health Serv Res. 2005;5:51.

37. Daggy J, Lawley M, Willis D, et al. Using no-show modeling to improve clinic performance. Health Informatics J. 2010;16(4):246-259.

38. Horwitz LI, Moriarty JP, Chen C, et al. Quality of discharge practices and patient understanding at an academic medical center. JAMA Intern Med. 2013;173(18):1715-1722.

39. Vidyarthi AR, Katz PP, Wall SD, Wachter RM, Auerbach AD. Impact of reduced duty hours on residents' education satistfaction at the University of California, San Francisco. Acad Med. 2006;81(1):76-81

40. Coffey C, Kufta J. Patient-centered post-discharge appointment scheduling improves readmission rates. Paper presented at: Society of Hospital Medicine, Annual Meeting 2011; Grapevine, Texas.

41. Chang R, Spahlinger D, Kim CS. Re-engineering the post-discharge appointment process for general medicine patients. Patient. 2012;5(1):27-32

42. Haftka A, Cerasale MT, Paje D. Direct patient participation in discharge follow-up appointment scheduling. Paper presented at: Society of Hospital Medicine, Annual Meeting 2015; National Harbor, MD.

43. Stubbs ND, Geraci SA, Stephenson PL, Jones DB, Sanders S. Methods to reduce outpatient non-attendance. Am J Med Sci. 2012;344(3):211-219.

44. Kros J, Dellana S, West D. Overbooking Increases Patient Access at East Carolina University's Student Health Services Clinic. Interfaces. 2009;39(3):271-287.

45. Hills LS. How to handle patients who miss appointments or show up late. J Med Practice Management. 2009;25(3):166-170.

46. Price H, Waters AM, Mighty D, et al. Texting appointment reminders reduces 'Did not attend' rates, is popular with patients and is cost-effective. Int J STD AIDS. 2009;20:142-144.

47. Parikh A, Gupta K, Wilson AC, Fields K, Cosgrove NM, Kostis JB. The effectiveness of outpatient appointment reminder systems in reducing no-show rates. Am J Med. 2010;123(6):542-548.

48. Shah SJ, Cronin P, Hong CS, et al. Targeted reminder phone calls to patients at high risk of no-show for primary care appointment: A randomized trial. J Gen Intern Med. 2016;31(12):1460-1466 